\title{
Wireless Connected Smart Microsystems
}

\author{
Chien-Yuan Liu ${ }^{1}$ and Cheng-Yi Chen ${ }^{2 *}$ \\ ${ }^{1}$ Department of Computer Science and Information Engineering, Cheng-Shiu University, \\ No. 840, Chengcing Rd., Niaosong Dist., Kaohsiung 83347, Taiwan \\ ${ }^{2}$ Department of Electrical Engineering, Cheng-Shiu University, \\ No. 840, Chengcing Rd., Niaosong Dist., Kaohsiung 83347, Taiwan
}

(Received August 1, 2017; accepted January 23, 2018)

Keywords: IoT, smart device, web, HTTP

The era of the Internet of things (IoT) has come. The goal of the IoT is to realize smart living for humans. In smart home applications, smart devices and control servers are exchanging information over networks. Traditionally, various IoT providers define different standards that apply to their own ecosystems. In this paper, we propose the use of a web model to realize the information exchange between smart devices and control servers. In this way, systems can easily communicate with each other through a unified form of IoT.

\section{Introduction}

The Internet of things (IoT) consists of a lot of smart devices, networks, and control servers with powerful computing capability. The goal of the IoT is to realize smart living for humans. Smart devices control sensors and actuators via microcontrollers. In general, microcontrollers read sensing data to control servers via networks. Next, control servers perform advanced control computation and then issue related control commands. Finally, microcontrollers receive the commands from the servers and operate the actuators according to the commands. Smart devices and control servers exchange information over networks and protocols.

The scale of the IoT is tens or hundreds of times larger than that of the Internet. Therefore, the IoT will be the biggest development in the next decade, which seems to be the common consensus of the information and communication technology (ICT) industry. ${ }^{(1)}$ Under the IoT trend, more and more smart devices are being invented for smart living. ${ }^{(2,3)}$ In a review of technological applications, ${ }^{(4)}$ the IoT was depicted as "an open and comprehensive network of intelligent objects that have the capacity to auto-organize, share information, data and resources, reacting and acting in face of situations and changes in the environment". In another overview of ITU-T, ${ }^{(5)}$ the IoT was described as follows: "from the perspective of technical standardization, IoT can be viewed as a global infrastructure for the information society, enabling advanced services by interconnecting (physical and virtual) things based on existing and evolving interoperable ICT".

The IoT reference model ${ }^{(5)}$ classifies the IoT as being composed of four layers: the top application layer, the application support layer, the network layer, and bottom device layer.

*Corresponding author: e-mail: k0464@gcloud.csu.edu.tw http://dx.doi.org/10.18494/SAM.2018.1765 
Since the network layer has to connect to the Internet, it is better to support an Internet protocol (IP) network intrinsically. Currently, IEEE 802.3, IEEE 802.11, and 3G/4G are able to connect a local smart device to the Internet. IEEE 802.3 is tethered by a physical wire. Thus, it is costly to deploy the solution to many existing buildings. IEEE 802.11 , known as $\mathrm{WiFi}$, is a wireless link, has very high data rate, and is free for local networks. 3G/4G has the largest coverage, but is very costly. As for the device layer, there are various communication technologies to link all end nodes to concentrators or gateways. Table 1 compares the features of various wireless technologies.

Note that LoRa and NB-IoT are two of the latest technologies proposed to link end nodes to concentrators or gateways. The business policies of the organisations behind these technologies are unclear and their linking modules are still rare. Thus, they need to be observed continuously. As for the other five technologies described in Table 1, generally, power consumption, cost, and coverage range are the major concerns for most IoT applications. On the other hand, tablets and smartphones have already become the main operating devices for humans for IoT applications. Smart devices usually have no ZigBee, Z-Wave, and Tread interfaces. Therefore, a gateway device is required to connect smart devices to various nonbuilt-in wireless technologies. Still, there are many applications unable to use wall power. In this case, power consumption is a real constraint. WiFi and Bluetooth are standard in almost every smartphone. WiFi and Bluetooth consume more power than ZigBee, Z-Wave, or Thread, yet are suited to wall-powered applications.

The key to success is to deploy the right wireless technologies that will meet the requirements of the application and to avoid the temptation of trying to make one technology satisfy every requirement. ${ }^{(6)}$ Traditionally, various IoT providers specify various standards that apply to their own ecosystems. A wide range of IoT standards means that the data from various smart devices must use a common communication protocol in order to exchange information. The World Wide Web (WWW), which is served by the Hypertext Transfer Protocol (HTTP), is the most common information service interface available today. Almost all platforms are highly compatible with the HTTP request and response model. Therefore, it is logical to use this protocol for communication between devices from various IoT vendors. In this paper we propose to use the HTTP request and response model to realize information exchange between smart devices and control servers. The implementation of the proposed system proves that low-

Table 1

Features of various wireless technologies.

\begin{tabular}{lcccccc}
\hline Technology & Security & $\begin{array}{c}\text { Power } \\
\text { consumption }\end{array}$ & Cost & Range & Data Rate & $\begin{array}{c}\text { Topology } \\
\text { flexibility }\end{array}$ \\
\hline WiFi & $\mathrm{H}$ & $\mathrm{H}$ & $\mathrm{M}$ & $\mathrm{M}$ & $\mathrm{H}$ & $\mathrm{M}$ \\
ZigBee & $\mathrm{H}$ & $\mathrm{L}$ & $\mathrm{L}$ & $\mathrm{L}$ & $\mathrm{M}$ & $\mathrm{H}$ \\
Bluetooth & $\mathrm{H}$ & $\mathrm{L}$ & $\mathrm{L}$ & $\mathrm{S}$ & $\mathrm{H}$ & $\mathrm{L}$ \\
Z-wave & $\mathrm{H}$ & $\mathrm{L}$ & $\mathrm{L}$ & $\mathrm{M}$ & $\mathrm{M}$ & $\mathrm{H}$ \\
Thread & $\mathrm{H}$ & $\mathrm{L}$ & $\mathrm{M}$ & $\mathrm{L}$ & $\mathrm{M}$ & $\mathrm{H}$ \\
LoRa & $\mathrm{H}$ & $\mathrm{M}$ & $\mathrm{X}$ & $\mathrm{L}$ & $\mathrm{L}$ & $\mathrm{H}$ \\
NB-IoT & $\mathrm{H}$ & $\mathrm{H}$ & $\mathrm{X}$ & $\mathrm{L}$ & $\mathrm{M}$ & $\mathrm{H}$ \\
\hline
\end{tabular}

H, high; M, medium; L, low or long; S, short; X, to be defined. 
cost single-board microcomputers can realize the WWW service for IoT. Moreover, the system can intuitively integrate smart devices at the endpoints of the IoT.

\section{System Analysis and Design}

To understand the technological suitability for smart living, the experiments proposed 3 types of wireless communications for IoT applications, which would cover most applications for smart living. The first one is a Bluetooth scenario, the second one is a ZigBee scenario, and the third one is a WiFi scenario.

The Bluetooth scenario: There are now many such applications in our daily lives. For example, a smartphone plays music to a Bluetooth speaker or gets data from Bluetooth weight meter. In these applications, Bluetooth is a better selection because of its low power consumption and low cost, and is pervasive in all tablets and smartphones. Bluetooth Low Energy (BLE) has emerged as a strong low-power wireless technology for single-hop communication use cases, which may enable the connection of a very large number of new devices to the $\operatorname{IoT}^{(7,8)}$

The ZigBee scenario: Home automation is the representative application. In home automation, there are many battery-powered sensors deployed around the home. Low power usage and multi-hop communication are essential because these sensors need to work for years. In this type of application, ZigBee, Z-Wave, and Thread are better selections since these technologies have a mesh topology and a relatively low power compared with others. However, popular tablets and smartphones do not support these interfaces directly. Therefore, a gateway is required to bridge the gap between smartphones and these wireless interfaces.

The WiFi scenario: There are many such applications in our daily lives too. For instance, via an access point (AP), a smartphone controls home lighting and a movie being played through a connected TV at the same time. In these applications, WiFi is a better selection because it is of high bandwidth and has multiple virtual links, and is also pervasive in all tablets and smartphones.

\subsection{Bluetooth scenario}

To show one of the applications in our daily lives, a smart limb-strength promoter system ${ }^{(9)}$ was designed as a demonstration in this case. The system is composed of two devices. The first is a smart stretch-sensing device, and the second is a smartphone preinstalled with a limbstrength promoter APP (mobile application program). The proposed system is shown in Fig. 1. Bluetooth is utilized as the link between the device and the smartphone. The performance of the system showed that limb stretching exercise is correctly sensed by the smart stretch-sensing device and the stretching count is properly shown on the display of the smart APP.

\subsection{ZigBee scenario}

To show one of the applications in smart schools, a promotion system for classroom interactions (PSCI) ${ }^{(10)}$ was designed as a demonstration in this case. The system is composed 



Fig. 1. (Color online) The smart limb-strength promoter system.

of three devices. The first is a keypad (KP). One KP is allocated to each student upon the beginning of the class to allow students to express their opinion. The second is a smart pad preinstalled with a chairing APP, which would manage the question and answer activity given by a teacher. The third device is a forwarding module (FM) to bridge the ZigBee network for the many KPs in a classroom and the Bluetooth interface in the smartphone. The proposed system is shown in Fig. 2. The performance of the system showed that the teaching and learning interactions could be supported by the system, e.g., the questions could be successfully dispatched to every individual student KP, the answers of all students could be correctly returned to the teacher's APP via pressing the keyboard on the student KP. The virtual channel built by the forwarding gateway could properly chain both sides into a group, and the result could be correctly calculated and shown by the APP.

\subsection{WiFi Scenario}

To demonstrate the WiFi applications in smart living, an APP for children companion devices and an APP for home control devices were proposed. The APPs are executed on a smartphone. Via WiFi, the smartphone can display the temperature or humidity in the children's room and enable remote adjustment of the air conditioner settings by the parent, as well as viewing the children's activity. The proposed system is shown in Fig. 3. WiFi is utilized as the link between the devices and the smartphone or smart pad. The features of the scenario are that multiple virtual links exist simultaneously and some of them work at a high data rate.

The child companion APP (CCAPP) and home control APP (HCAPP) will be implemented by JavaScript with node.js in the Android system. The CCAPP and HCAPP periodically obtain sensor data from or send control commands to the home control firmware run in Arduino and to the children companion APP run in Raspberry Pi, respectively. HCAPP also receives video streams from the Raspberry Pi if the camera viewing function is opened by the parents. The system architecture is shown in Fig. 4. All the communication interfaces will be exchanged via the web. Through Restful HTTP request and reply, the web client APP can receive or send data from or to the web server. ${ }^{(1)}$ Figure 4 shows the software architecture of the smart living application system. 


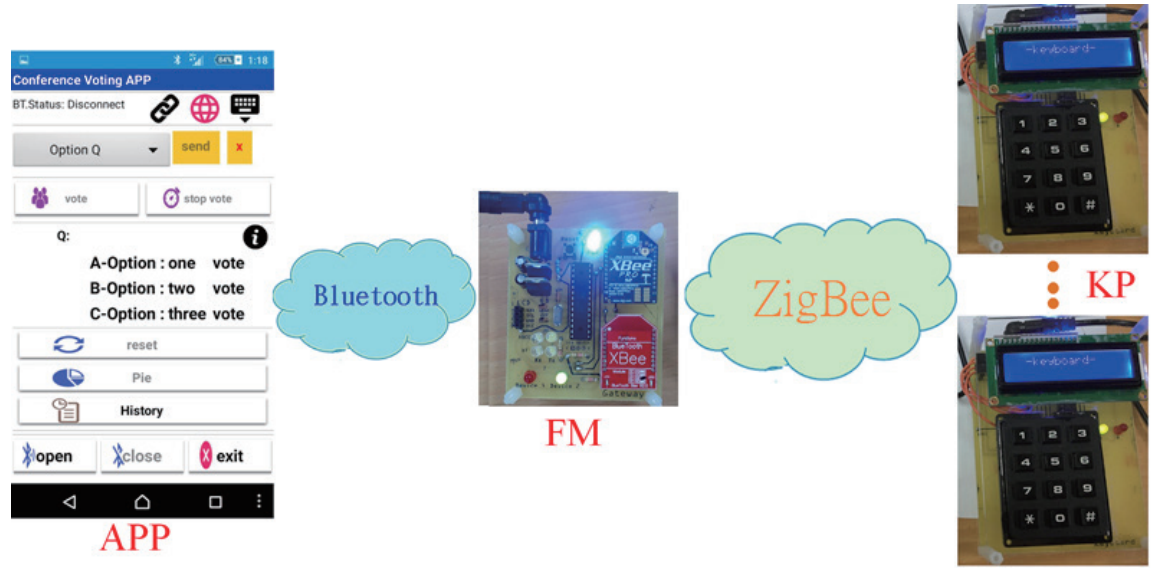

Fig. 2. (Color online) Promotion system for classroom interactions.



Fig. 3. (Color online) Smart living applications via WiFi.

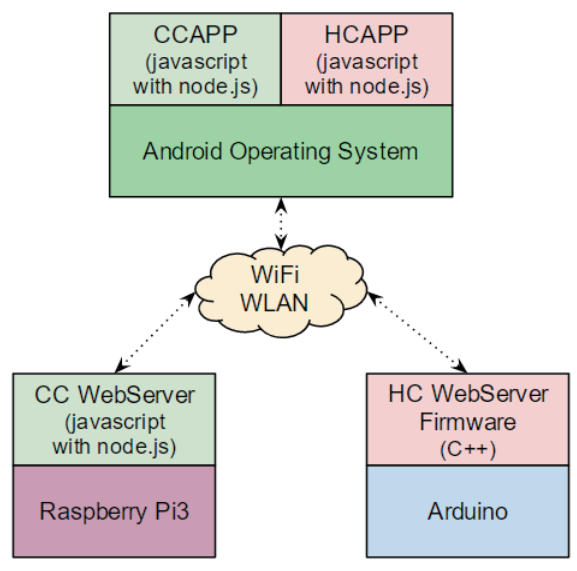

Fig. 4. (Color online) Software architecture for WiFi-based smart living.

\section{Conclusions}

This article presents the analysis, design, and implementation of smart device information exchange. Three application scenarios with various communication protocols are described: the Bluetooth communication scenario, which is usually applied to the link of a pair of devices; the ZigBee communication scenario, which is typically utilized by links between a group of devices; and the WiFi communication scenario, which is generally adopted by group communications mixed with a high bandwidth requirement. The systems implemented for the former two scenarios are completed and correctly verified. Regarding the last scenario, the system application and the software architecture are only proposed, and are still under development. In the future, the application programs on three different platforms, i.e., Android, Arduino, and Raspberry $\mathrm{Pi}$, will be implemented and validated according to the proposed architecture of the last scenario. 


\section{Acknowledgments}

The authors thank Mr. Yan-Zhe Su for assistance with implementation.

\section{References}

1 O. Vermesan and P. Friess: Internet of Things: Converging Technologies for Smart Environments and Integrated Ecosystems (River Publishers, Aalborg, Denmark, 2013).

2 S. Kumar and S. R. Lee: Proc. 18th IEEE Int. Symp. Consumer Electronics (ISCE 2014) 1.

3 R. Das, G. Tuna, and A. Tuna: Int. J. Comput. Networks Appl. 2 (2015) 242.

4 S. Madakam, R. Ramaswamy, and S. Tripathi: J. Comput. Commun. 3 (2015) 164.

5 International Telecommunication Union (ITU): Internet of Things Global Standards Initiative, http://www.itu. int/en/Pages/default.aspx (accessed July 2017).

6 N. Baker: Comput. Control Eng. 16 ( 2005) 20.

7 C. Gomez, J. Oller, and J. Paradells: Sensors 12 (2012) 11734.

8 K. H. Chang: IEEE Wireless Commun. 21 (2014) 6.

9 C. Y. Liu: ICIC Express Lett. 7 (2016) 237.

10 C. Y. Liu and C. Y. Chen: ICIC Express Lett. Part B 7 (2016) 2505.

11 M. Huynh and P. Ghimire: J. Inf. Technol. Educ.: Innovations Pract. 14 (2015) 145.

\section{About the Authors}

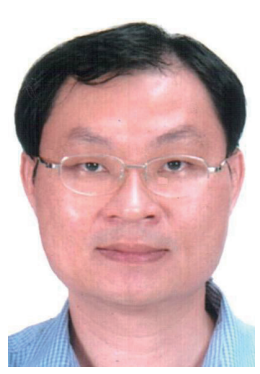

Chien-Yuan Liu received his B.S. degree from the Department of Electrical Engineering of the National Taiwan Institute of Technology in 1983, his M.S. degree from the Department of Electrical Engineering in the National Sun Yat-Sen University, Taiwan in 1992, and his Ph.D. degree from the Department of Computer Science and Engineering of the National Sun YatSen University in 2005. From 1985 to 2000, he worked as an engineer in the process control computer division of China Steel Corporation, Taiwan. Since August 2000, he has been at Cheng Shiu University. His research interests are in IoT and smart systems. Since 2012 he has been an associate professor at Cheng-Shiu University.

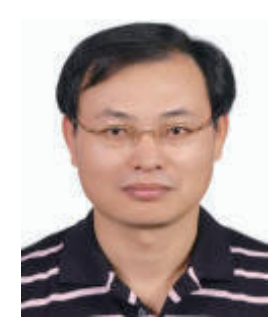

Cheng-Yi Chen received his M.S. degree from the Department of Mechanical Engineering of the National Sun Yat-Sen University, Taiwan in 1994, and his Ph.D. degree from the Department of Mechanical Engineering of the National Sun Yat-Sen University in 1999. From 1999 to 2001, he was an associate professor of the Department of Automation Engineering of the Kao Yuan institute of Technology. From 2001 to 2009, he worked as an associate professor of the Department of Electrical Engineering in Cheng Shiu University. Since July 2009, he has been a professor in Cheng Shiu University. His research interests are in nonlinear control, automation control, motion control, embedded control system design, and smart systems. 